Castrejon-Pita, J.R., Castrejon-Garcia, R., and Hutchings, I.M., 'High speed shadowgraphy for the study of liquid drops', in Klapp, J., Medina, A., and Cros, A.-M. (Eds) 'Fluid Dynamics in Physics, Engineering and Environmental Applications', Springer, 2012 ISBN-10:

3642277225 


\title{
High speed shadowgraphy for the study of liquid drops
}

José Rafael Castrejón-Pita, Rafael Castrejón-García ${ }^{\S}$ Ian Hutchings

Department of Engineering, University of Cambridge, CB3 0FS, 17

Charles Babbage Road, Cambridge, United Kingdom.

${ }^{\S}$ Centro de Investigación de Energía, UNAM, Priv. Xochicalco s/n, Te-

mixco, Morelos, 62580, México.

E-mails: jrc64@cam.ac.uk, rcg@cie.unam.mx, imh2@cam.ac.uk

\begin{abstract}
The principles of shadowgraph photography are described in this work together with a few examples of its utilisation in the study of free liquid surfaces. Shadowgraph photography is utilized in combination with high speed imaging and image analysis to study the behaviour of sub-millimetre and millimetre-sized droplets and jets. The temporal and physical scales of these examples cover operational ranges of industrial, commercial, and academic interest. The aim of this work is to summarize the necessary optical and illumination properties to design an appropriate shadowgraph imaging system.
\end{abstract}

\section{Introduction}

Over the last two centuries, the shadowgraph technique has been extensively used in the study of fluid dynamics, the visualisation of objects in motion, in optical microscopy and in scientific photography. In fact, it can be said that several scientific breakthroughs could not have been achieved without this technique. Perfect examples of these are the early experiments in fluid mechanics carried out by Savart and the study of the breakup of fluids by Raleigh, both in the nineteenth century (Savart, 1866 and Ray- 
leigh, 1879). Savart developed a primitive but effective shadowgraph setup to visualize liquid jets emerging from a pressurized nozzle and his results were used some years later by Rayleigh and Plateau to lay the theoretical basis that describes the natural break up of jets (Eggers and Villermaux, 2008). In simple terms, shadowgraphy is a visualisation technique in which the observed objects are illuminated from behind. Shadowgraph photography is often used to visualize liquids, but can also be used to observe solid objects and gases. Shadowgraphy is an advantageous visualisation technology due to its simplicity, low cost, quality and by the fact that a large range of optical components are readily commercially available. Furthermore, thanks to the advances in digital imaging, shadowgraphy is no longer a qualitative technique as the digitalisation of images and their process can provide information about the dynamics of the object studied (Castrejón, Rev. Mex. Fis. 2011).

Shadowgraphy has a large range of potential applications: it is used in aerodynamics, in fluid dynamics and in ballistics. Although extensively used and mentioned in the scientific literature, the theoretical aspects of the technique are not generally discussed in detail or even generally available. As a result, the design and construction of shadowgraph systems is usually difficult and often carried out empirically. The aim of this work is to present and discuss the optical and technical concepts involved in shadowgraph photography, in particular the exposure time, field of view and depth of field. Several shadowgraph images of sprays and droplets are shown to exemplify the capabilities of the shadowgraph method for the study of fluids. These systems differ in their temporal and physical scales, components, and in the way they are designed and constructed; from custom-built electronics and optics to commercially available cameras, flash-lamps and lenses.

\section{Shadowgraph illumination}

Shadowgraph photography or shadowgraphy is de facto a very simple technique that requires the optimisation of several parameters to obtain satisfactory results. Commonly, in conventional photography, the illumination of an object can be carried out in two ways: by front-illumination or by back-illumination. In the first case, illustrated in Fig. 1, the object is illuminated in such a way that the light dispersed or reflected by it forms the image on the camera sensor. This corresponds to conventional photography where a light source is always positioned in front of the object. This 
type of illumination is natural and necessary for the perception of colour in photography and in the image formation in the eye.

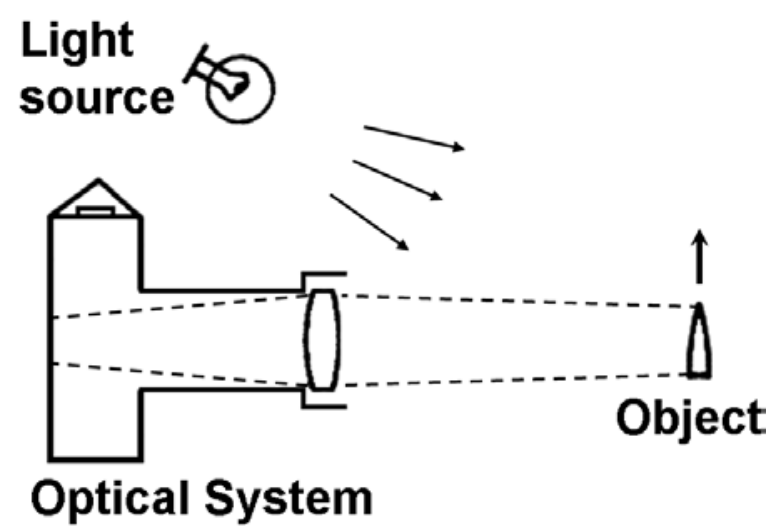

Figure 1 Front illumination scheme, usually used in conventional photography.

In the second case, illustrated in Fig. 2, the object is illuminated from behind by means of a focusing lens in such a way that the object's image formed on the sensor camera corresponds to its shadow or silhouette. On the recorded image, the object's shadow contrasts with a bright field formed by the light from the illumination system. This technique is known as Shadowgraphy. This type of illumination does not usually record colour information as the light that interacts with the object is mostly refracted out of the sensor. However, this technique captures most of the light coming from the source and, as a result, is ideal in conditions of low light or with sensors with low sensitivity. This characteristic renders the shadowgraph technique the preferred type of visualisation in high speed imaging where the sensitivity is low at high frame rates. Due to this fact, shadowgraphy is once again becoming widely used. Examples of front and back (shadowgraph) illumination are presented in Figures 3 and 4.

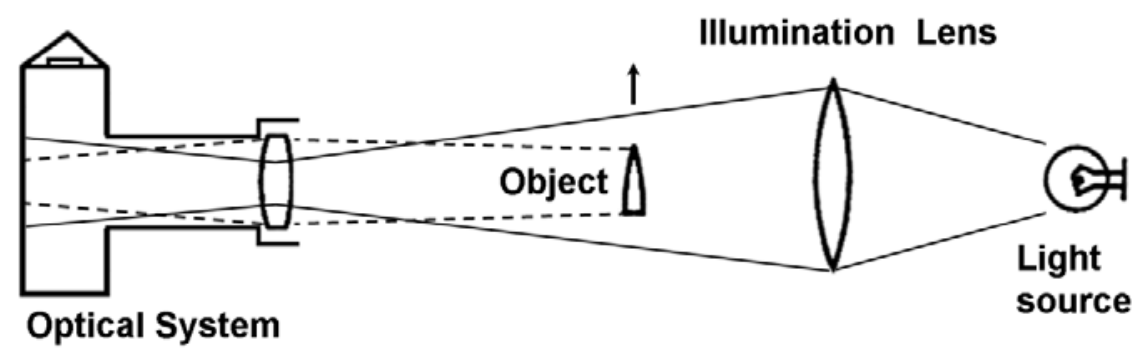

Figure 2 Shadowgraph illumination. 
Figure 3 shows a sequence of images in which a sessile colourless droplet is being impacted by a coloured one. The impacting droplet is travelling at $1.1 \mathrm{~m} / \mathrm{s}$ and has a radius of $1 \mathrm{~mm}$. Both droplets consist of a 75\% glycerine and water mixture with a viscosity of $100 \mathrm{mPa}$ s. The substrate is a flat transparent piece of acrylic sheet (polymethyl methacrylate). In this case, the illumination system is similar to that presented in Figure 1, where the light source (a $50 \mathrm{~W}$ filament lamp) is oblique to the object and placed in front of it. For these experiments, a Phantom V640 high speed camera was used to capture the impact at a rate of 1000 frames per second (fps) and at an exposure time of $0.3 \mathrm{~ms}$.
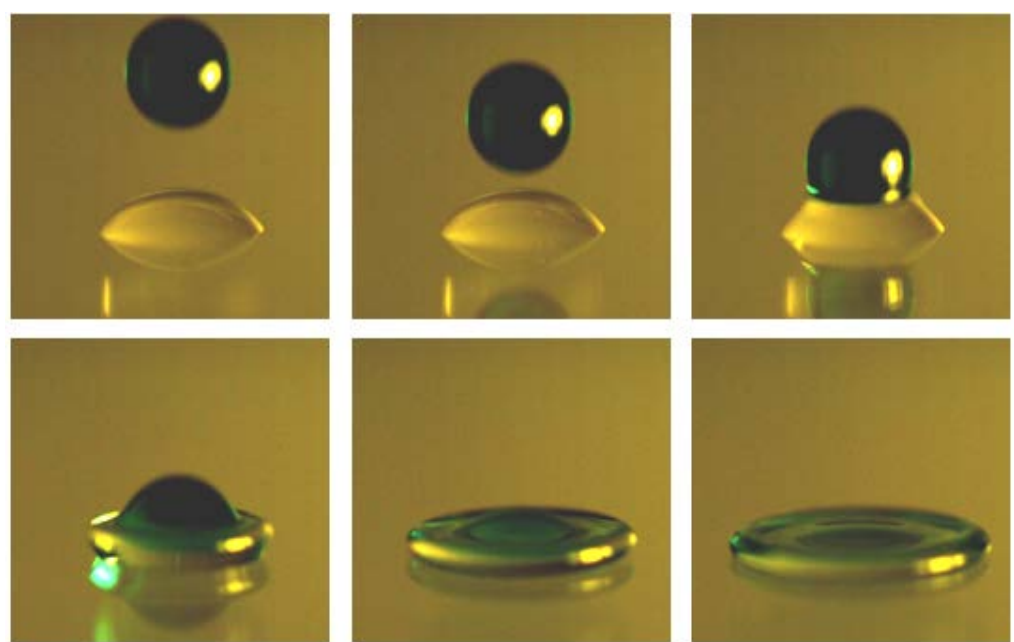

Figure 3 Examples of high speed imaging using a front illumination scheme; the images correspond to a sequence of images with a $1 \mathrm{~ms}$ separation. In this case, only the light that interacts with the droplets is collected by the lens and the sensor of the camera allowing the production of colour. In this scheme, the reflected image of the light source often appears as a bright spot

Figure 4 shows the impact of a droplet into a sessile one (same conditions as in Fig. 3) but now with the illumination being produced from the back (i.e. shadowgraph). The uniform background is created by an optical diffuser placed in front of the $50 \mathrm{~W}$ lamp. For these experiments, a V310 Phantom camera was used at a frame rate of 7000 fps and with exposure times of $2 \mu$ s (only images at equivalent times to those in Fig. 3 are shown). The exposure time and the frame rate in these two experiments were chosen to acquire images with similar brightness and contrast. In high speed imaging, the brightness of the images depends mostly on the frame rate; the faster the frame rate is, the less the time there is to collect 
photons on the sensor. As a consequence, to create clear images, there is always a compromise between frame speed and brightness. In fact, in most high speed imaging experiments, the illumination is the principal factor limiting the speed of recording. Both these cameras (the V310 and the V640) share the same CCD (the Phantom 1280x800 CMOS sensor) and sensitivity.
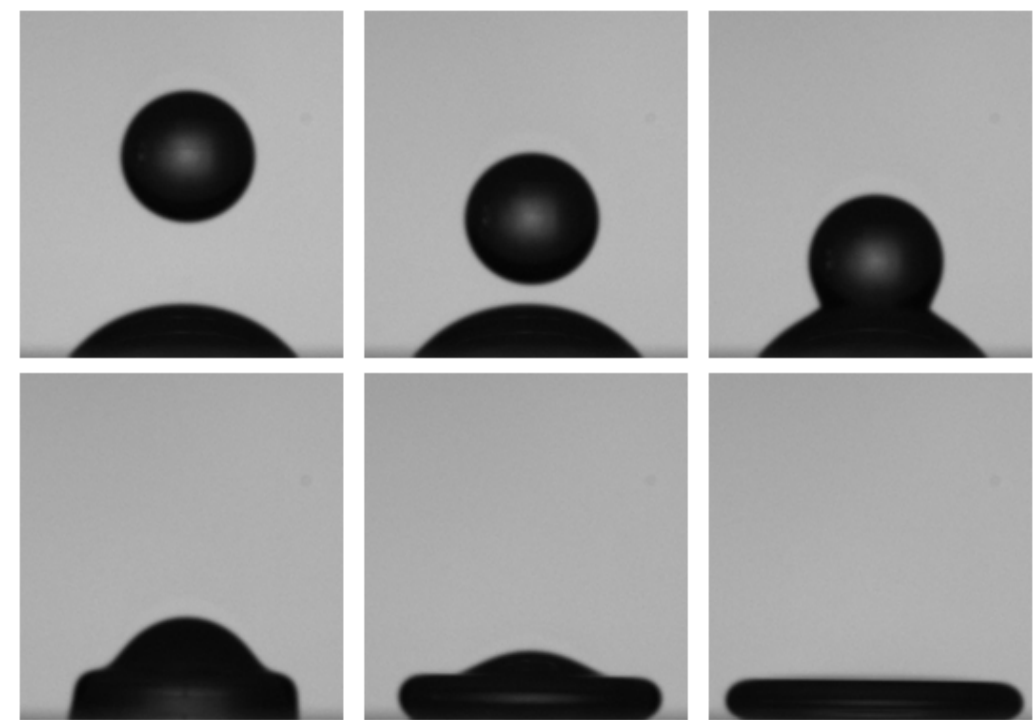

Figure 4 The same experiments as in Fig. 3, but with shadowgraph illumination. In this case, only the light that does not interact with the droplets is captured by the sensor. All the other rays are mostly either reflected or refracted out of the camera lens. The brighter region at the center of the spherical drop is caused by light refracted through the drop that is not directed outside the CCD sensor.

The frame speed is different between the images presented in Figs. 3 and 4 (front and shadowgraph imaging) as it was adjusted to cope with the different type of illumination. The recording under shadowgraph conditions can be faster and with shorter exposure times due to a more efficient illumination. This is a useful characteristic of shadowgraphy as shorter exposure times are usually desired in the study of object in motion in order to avoid the recording of blurred images. This experimental approach has been used in droplet deposition studies to validate theoretical models and to investigate the inner fluid dynamics during the deposition and coalescence of impacting droplets, (Castrejón-Pita et al., 2011).

Nowadays, the use of image analysis methods is becoming increasingly popular in studies of fluid dynamics. For the correct recognition of fluid or 
object boundaries, most image analysis algorithms require the objects to be sharp focused, frozen in time, and visualized on an even and homogenous background. These characteristics are determined by the properties of the optical elements and the illumination arrangement. For objects in motion, the image sharpness is determined by the exposure time of the recording sensor and by the depth of field and focus of the optical system. In most cases, the blurring due to the motion is controlled by the use of short exposure and shutter times, or short light pulses. For example, let us assume that an object is moving at a speed of $50 \mathrm{~m} / \mathrm{s}$. If the object is observed through an optical system with a magnification factor of 3 , the velocity of object's image on the camera sensor is actually $150 \mathrm{~m} / \mathrm{s}$. If the shutter time is set to $100 \mu \mathrm{s}$, the image would appear blurred on the sensor over a distance of $15 \mathrm{~mm}$. On the other hand, if the object is moving at $1 \mathrm{~m} / \mathrm{s}$, the object would appear blurred only along $0.3 \mathrm{~mm}$. In words used in conventional photography, the shutter time must be short enough to "freeze" the motion of the object. This effect is actually observed in Figures 3 and 4. In Figure 3, the impacting droplet appears blurred along the axial direction for about one third of its radius. On the contrary, in Figure 4, as the exposure time is shorter (150 times faster), the image is sharper, and its contour well-defined and perfectly circular whereas the image of the droplet in Fig. 3 has an elliptical shape due to the blurring.

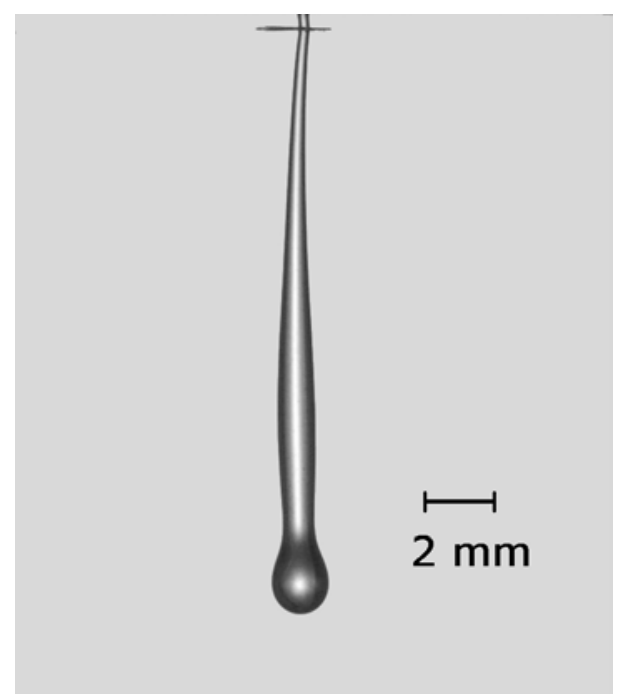

Figure 5 Shadowgraph snapshot of a $0.5 \mathrm{~m} / \mathrm{s}$ droplet of a $83 \%$ glycerine and water mixture. A Nikon SB-800 flash was used to produce the $24 \mu$ s pulse used to capture this image. 
The shutter times of most commercial single-lens reflex cameras (SLR cameras) are rarely below $500 \mu$ s, which is the key parameter to consider when considering photographing objects in motion with conventional CCD cameras. In contrast, in high speed imaging, exposure times are usually in the range of $1 \mathrm{~ms}$ to $200 \mathrm{~ns}$ (subject to a change in resolution).

Alternatively, the use of a flash lamps or pulsed light source is recommended where the pulse duration is shorter than the shutter time. In the latter case, the effective exposure time is determined by the light duration and not restricted to the shutter time. Spark flashes or xenon flash lamps are the best options for shadowgraphy. Photographic flashes are readily commercially available, are not expensive, have a pulse duration in the range of 20 to $200 \mu \mathrm{s}$ and are viable for low speed applications. These flashes are commonly used to study the jetting and behaviour of millimetre size droplets, (Castrejón-Pita et al, 2008). An example of this is shown in Figure 5.

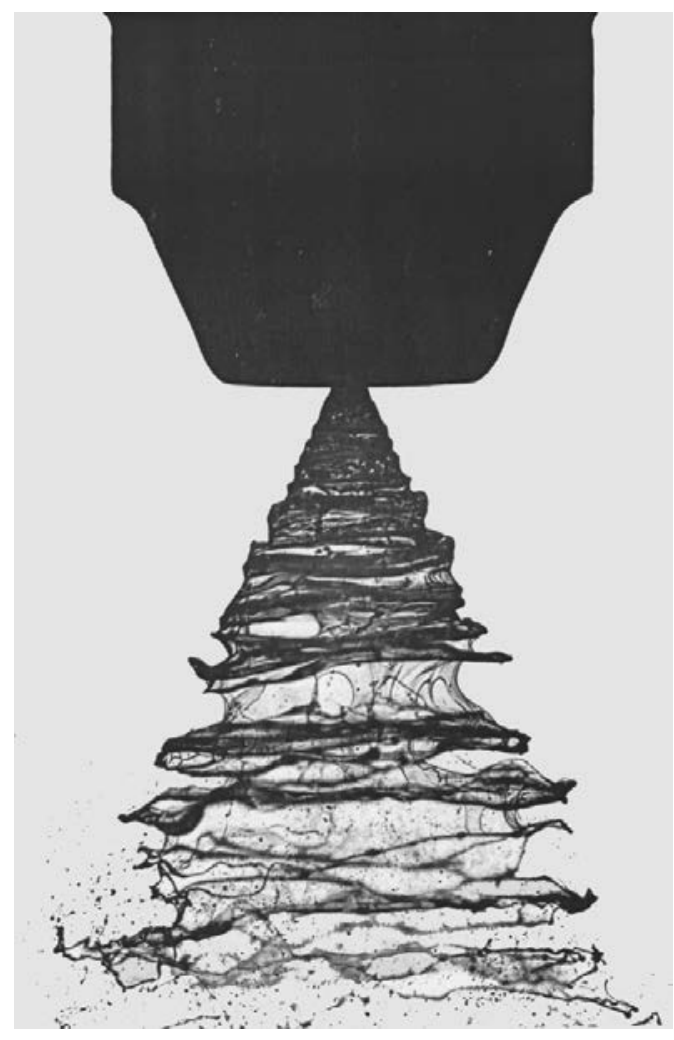


Figure 6 A shadowgraph snapshot of an oil spray. A 300 ns spark flash system was used for this illumination. The nozzle output has a diameter of $2 \mathrm{~mm}$ and the droplet terminal speed is $15 \mathrm{~m} / \mathrm{s}$.

Laboratory xenon and spark lamps produce much faster light pulses but operate with high voltages which may restrict their application, based on safety issues, especially when used in studies involving volatile, conductive or explosive fluids. Specialist xenon systems produce light pulses with durations ranging from $120 \mathrm{~ns}$ to $1 \mu \mathrm{s}$ with $100-200 \mathrm{~mJ}$ of pulse energy. In contrast, spark flashes have a pulse light duration of a few nanoseconds. These setups generally consist of a pair of electrodes connected to a capacitor which is charged until the stored energy is released as a luminous spark. The light intensity and the pulse duration can be adjusted by varying the separation and size of the electrodes and the surrounding medium. Many of the commercially available systems use argon gas, and produce pulse durations of the order of 5 to $20 \mathrm{~ns}, 9$ to $25 \mathrm{~mJ}$ of electric flash energy, and utilize 3- $5 \mathrm{kV}$ power supplies. Although expensive, these systems are in many cases very convenient as they require little maintenance. Figure 6 shows an example of the use of a spark flash system to image a fast spray generator; this technology has been used in the past to study the fractal dimension of the aerosol formed and in the study of drop size distributions in sprays, (Castrejón-García et al, 2003) and (Le Moyne et al, 2008).

The exposure time is only one of the factors determining the sharpness of the objects in an image. Other important parameters are the depth of field and the working distance of the optical system used to visualize the objects. The correct implementation of shadowgraph imaging requires the understanding not only of the individual components of the imaging setup but also their overall effect on the process of image formation. In most cases a few concepts of optics, ray tracing, and photography are needed to set up a suitable working system. The following section presents the theoretical basis required to design and implement a working shadowgraph scheme.

\section{Image formation and object illumination}

As mentioned above, the aim of shadowgraph photography is to obtain a sharp or focused silhouette of an object on an even or homogenous background. To fulfil these conditions, any shadowgraph system should contain an appropriate optical system capable of producing a focused image of the object on the plane of the optical sensor (i.e. film or CCD) whilst produc- 
ing simultaneously a uniformly illuminated background. One of the best ways to understand and visualize the operation of many optical systems is by drawing ray diagrams. In agreement with geometrical optics, any shadowgraph setup contains two sets of optics, one for the illumination and another one for the imaging of the object. Figure 7 represents a simplified setup consisting of two lenses, the object, and a plane where a camera sensor is placed. The illumination lens, labelled $\mathrm{L}_{\mathrm{i}}$ in the figure, is placed between the light source and the object. The imaging lens $L_{p}$ is located between the object and the sensor plane, and lens $L_{i}$ focuses the image of the light source on the nodal plane of $\mathrm{L}_{\mathrm{p}}$. The converging illumination rays from $L_{i}$ intercept the plane of the object focused by $L_{p}$. This implies that all the rays coming or passing through a particular point on the focused plane are collected by the lens $L_{p}$ and form a corresponding point of the image on the camera sensor. In other words, all the rays emitted or reflected by an object placed on the focused plane form the object's image on the camera sensor plane. If the object is only illuminated from its back, as in shadowgraphy, the image formed on the camera plane corresponds to its silhouette or shadow. It must be borne in mind that the homogeneous illumination field is produced only by the rays converging to the camera sensor plane (shaded area in Fig. 7). Therefore, the camera sensor must be smaller than the area illuminated by these rays; otherwise, the illumination field would not be homogeneous or uniform.

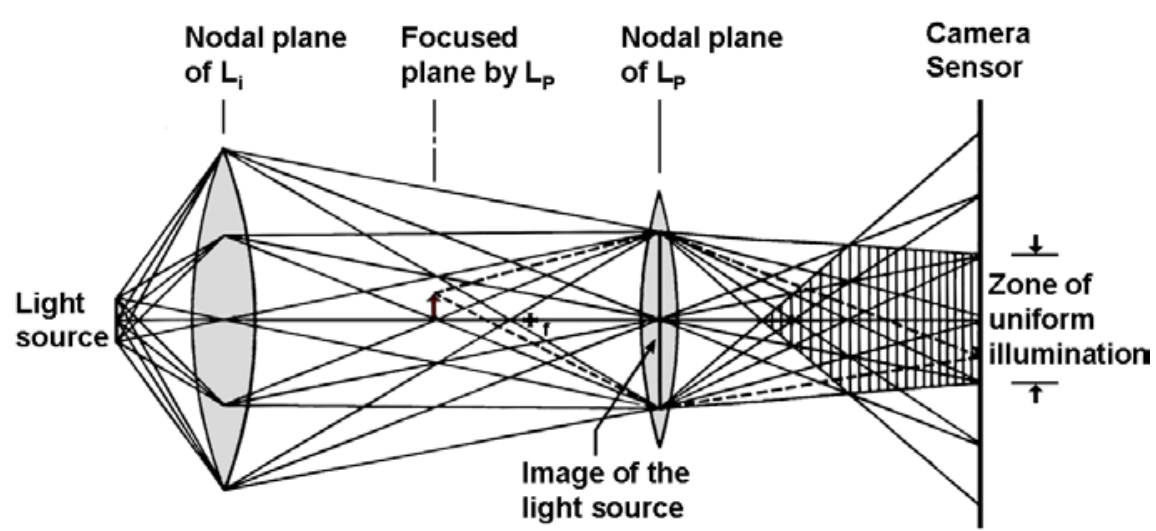

Figure 7. Ray tracing diagram illustrating the formation of an image in the shadowgraph technique.

A quick method to assess the size and position of the area of uniform illumination is to mount the camera lens without the camera body and form the image on a diffusive surface, e. g. a sanded piece of acrylic or glass. As 
optical components are usually more readily available than camera sensors this methodology is often useful to select a particular camera sensor.

\section{Depth of field of shadowgraph systems}

The depth of field is an important parameter in the specification of a photographic system, as it quantitatively defines the observation volume or measuring volume of a given setup. The depth of field is defined as the region surrounding the focused plane (forward and backwards) where the loss of sharpness is negligible or imperceptible. This loss of sharpness is gradual and increases as the object moves away from the focused plane; therefore, is not possible to determine a given depth of field unless a fixed loss-of-sharpness is set. In conventional photography the depth of field is given by (Lambrecht and Woodhouse, 2011):

$$
d_{F}=2 c \frac{f}{D}\left(\frac{m+1}{m^{2}}\right),
$$

where $f$ is the focal length of the lens, $m$ is the magnification of the optical system, $D$ is the lens diameter and $c$ is the so called circle of confusion, which is defined as the minimum loss-of-sharpness that is perceptible by the human eye. A typical value of the circle of confusion $(c)$ for the average human eye is (Highton, 2011):

$$
c=\frac{\text { inch }}{1000}=0.0254 \mathrm{~mm} \text {. }
$$

A circle or dot with size equal or smaller than $c$ will be seen as a point by the human eye at a comfort reading distance $(250 \mathrm{~mm})$. That is to say, if the blurring of an image is less or equal to $0.0254 \mathrm{~mm}$, the image is seen by the human eye as in perfect sharpness.

Equation (1) is correct for conventional photography but not necessarily valid for a shadowgraph system. Consider Fig. 8, where an object is placed a small distance $\delta u$ away from the plane focused by lens $\mathrm{L}_{\mathrm{p}}$. The lens diameter is $D$ and the object's image is formed at a distance $-\delta v$ from the CCD or film plane. The illumination lens $\mathrm{L}_{\mathrm{i}}$ is placed in such a way that the image $S$ of a circular light-source is formed in the nodal plane of the imaging lens. The rays that pass grazing the edge $\mathrm{E}$ of the object can enter $\mathrm{L}_{\mathrm{p}}$ only through the circle $S$ formed by the image of the light source. The limit rays 
that can enter $L_{p}$ are those coincident with points $s_{1}$ and $s_{2}$, and are separated by the angle $\beta$ that is responsible for the image blurring $\Delta$.

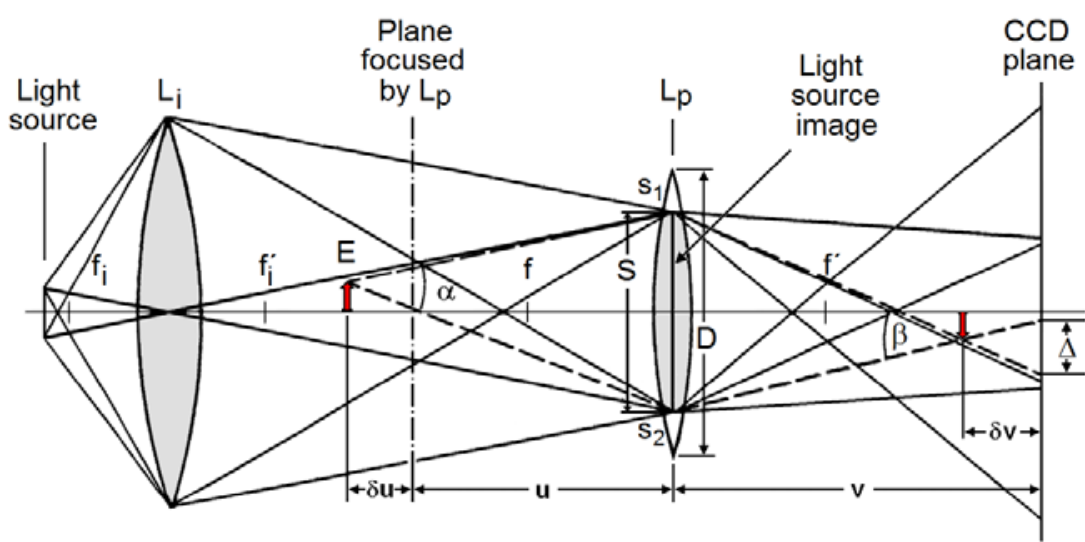

Figure 8 Ray diagram for an object placed outside the focused plane in a shadowgraph system.

As seen in Fig. 8, the smaller the size of $S$, the smaller the blur $\Delta$ and longer the depth of field of the optical system. Consequently, the depth of field of a given shadowgraph system only depends on the size $S$ of the image of the light source regardless of the diameter $\mathrm{D}$ of the lens $\mathrm{L}_{\mathrm{p}}$. The ray diagram in Fig 8 can be used to demonstrate that the depth of field in shadowgraph systems is (Castrejón-García, 1982):

$$
d_{F}=2 c \frac{f}{S}\left(\frac{m+1}{m^{2}}\right),
$$

which is identical to Eq. 1, with the exception that $S$ replaces $D$. Hence, the solely way to obtain a diaphragm-effect in a shadowgraph system to vary the depth of field is by changing the size of the image $S$ of the light source. Figure 9 shows a variable size light source to control the depth of field of a shadowgraph optical system by changing the size of the light source. Light from the spark flash is stopped by a pupil where a diffuser plate (sanded glass slide) is placed a given distance from the pupil. In position (a) near to the pupil, the diffuser works as a small light source, rendering a large depth of field; in position (b) the diffuser works as a larger light source, giving a short depth of field. Besides the device controlling the depth of field, the system also provides the same light energy, regardless of the position where the diffuser is placed. So there is no need to adjust other system parameters such as the sensitivity of the CCD sensor. 


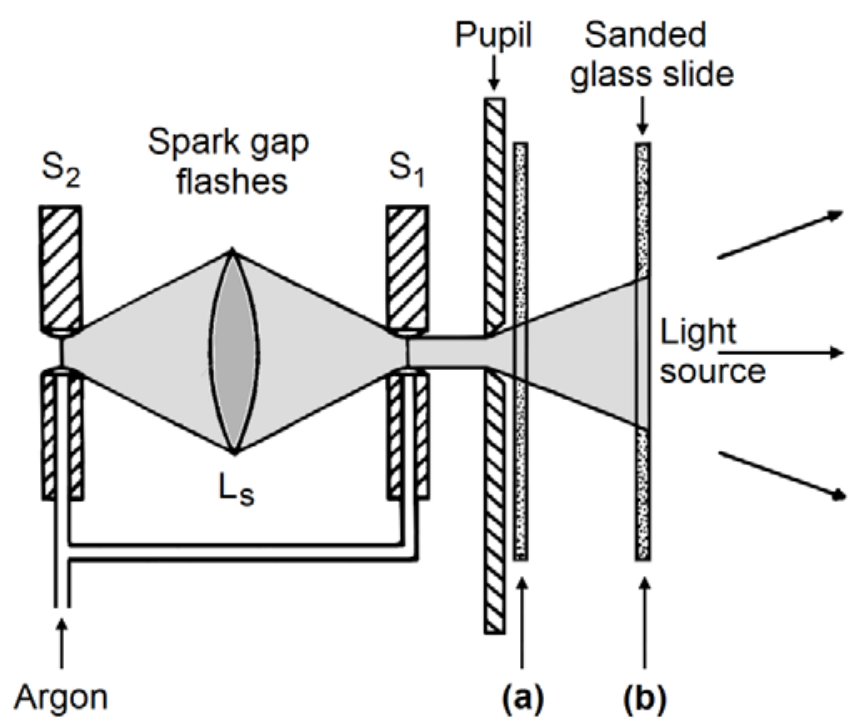

Figure 9 Schematic view of an optical arrangement to control the depth of field in a shadowgraph system.

\section{Applications of Shadowgraphy}

Shadowgraph systems have been used in experiments in fluid mechanics for many years. Currently, the technique is used in industrial processes to assess the behaviour of liquid dispensers, droplet generators, fuel injectors, turbine burners, and aerosols. In many of these cases, this technique produces quantitative data that can be analysed and used to control the efficiency of certain processes. In this section some examples of the application of shadowgraphy in industry are explained.

\subsection{Double-exposure shadowgraphy}

One of the most utilized and often cited shadowgraph setups is the double-exposure system (Jones, 1977), schematically shown in Fig. 10. As usual, the plane in which the object is moving is focused by the principal lens $\left(\mathrm{L}_{\mathrm{p}}\right)$ forming the object image on the camera sensor. The illumination is produced by two spark light sources, namely; first spark flash $\left(\mathrm{S}_{1}\right)$ and second spark flash $\left(\mathrm{S}_{2}\right)$. The lens $\left(\mathrm{L}_{S}\right)$ forms a real image of the second 
spark flash $\left(\mathrm{S}_{2}\right)$ on the position where the first spark flash $\left(\mathrm{S}_{1}\right)$ is placed. This way, the light emitted by both spark flashes, come optically from the same point. The illumination lens $L_{i}$ focuses the light produced by $S_{1}$ and $\mathrm{S}_{2}$ on the nodal plane of the imaging lens $\mathrm{L}_{\mathrm{p}}$, and is sent towards the sensor (CCD or film) to produce the illumination background. The illumination path is depicted by the gray coloured area in Fig. 10.

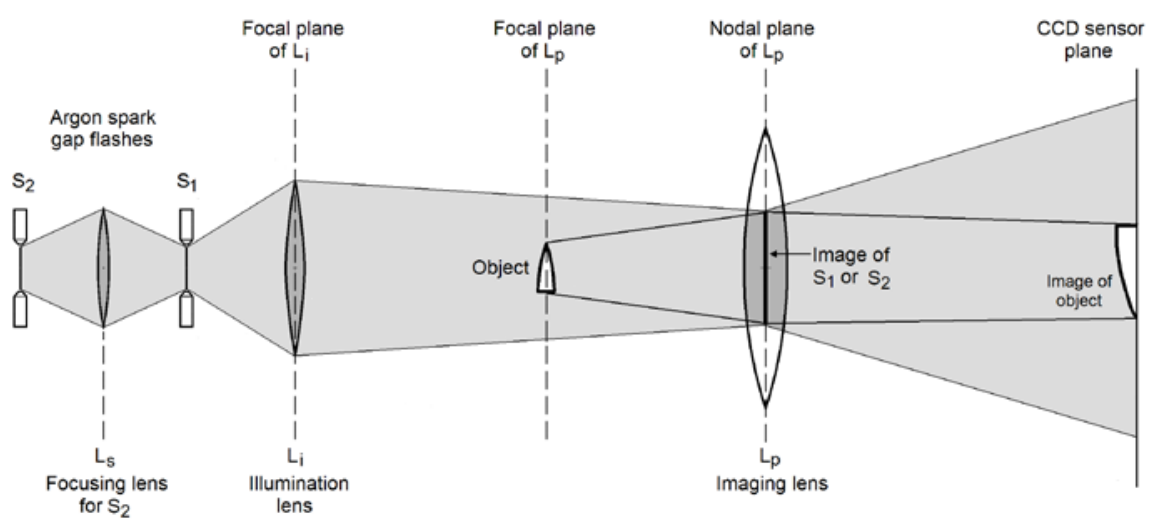

Figure 10 A scheme of a double-exposure shadowgraph system.

This system is known as a double exposure system because the light of each spark flash is recorded in the camera sensor on a single frame. The change of the position of the object's image $(\Delta r)$ is determined by the time between flashes $(\Delta t)$, the object speed $(v)$, and the magnification of the optical system $(m)$. Then, the actual velocity of the object is simply given by:

$$
v=\frac{\Delta r}{m \Delta t}
$$

Figure 11 shows a double-exposure shadowgraph image taken with an elapsed time $(\Delta t)$ between flashes set to $1 \mu \mathrm{s}$. The second image produced by the second flash appears darker than the one produced by the first flash. The evolution of ligaments and droplets from sprayed oil can be observed. 


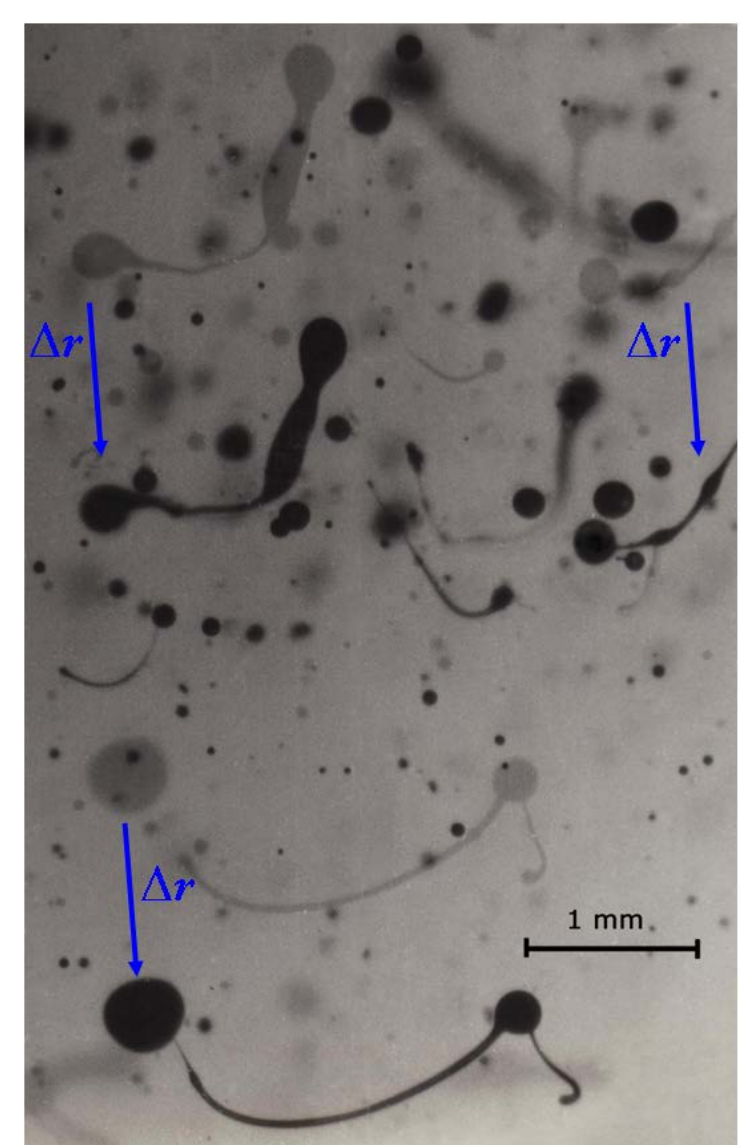

Figure 11 A double-exposure shadowgraph image of oil droplets and ligaments. The flash duration was 200 ns and the images were formed in a double spark flash rig.

\subsection{Shadowgraphy in the inkjet industry}

The shadowgraph technique is regularly used in the inkjet industry to monitor the delivery of fluid materials in a large variety of environments (Eggers and Villermaux, 2008). In fact, some laboratory-based inkjet printheads have built-in stroboscopic visualisation setups to inspect the behaviour of the jetted droplets used to create printed patterns, (i.e. Dimatix material printer DMP-2800). Although this capability is not yet and may never be available in other printers, this approach is usually carried out to test the performance of print-heads after their manufacturing. 
This section describes the use of a shadowgraph system to visualize a stream of high speed droplets generated by a commercially available continuous ink jet (CIJ) system by stroboscopic light. The setup utilized was a Domino Printing Sciences Ltd A-series single nozzle CIJ printer with a reported nozzle diameter of $60 \mu \mathrm{m}$. Essentially, in CIJ mode the liquid is pumped continuously into the head, generating an internal pressure which drives the liquid through the nozzle and creates a jet of the desired speed. The CIJ technology relies on the creation of a stream of droplets by the modulation (or periodic forcing) of a continuously running liquid jet, (Kalaaji et al, 2003 and Bruce, 1976). During the process of jetting, the jet can be charged by nearby electrodes set to a specific potential. After the jet break up the droplet is no longer electrically connected to the rest of the fluid and so the charge is retained by the droplet. The setting of the charging electrode determines the amount of charge induced to the droplet. The direction of individual drops is then controlled by a fixed electric field to form the printed pattern (Martin et al, 2008). The rate of droplet creation in these systems is equal to the frequency of the modulation that forces the breakup (Bruce, 1976).

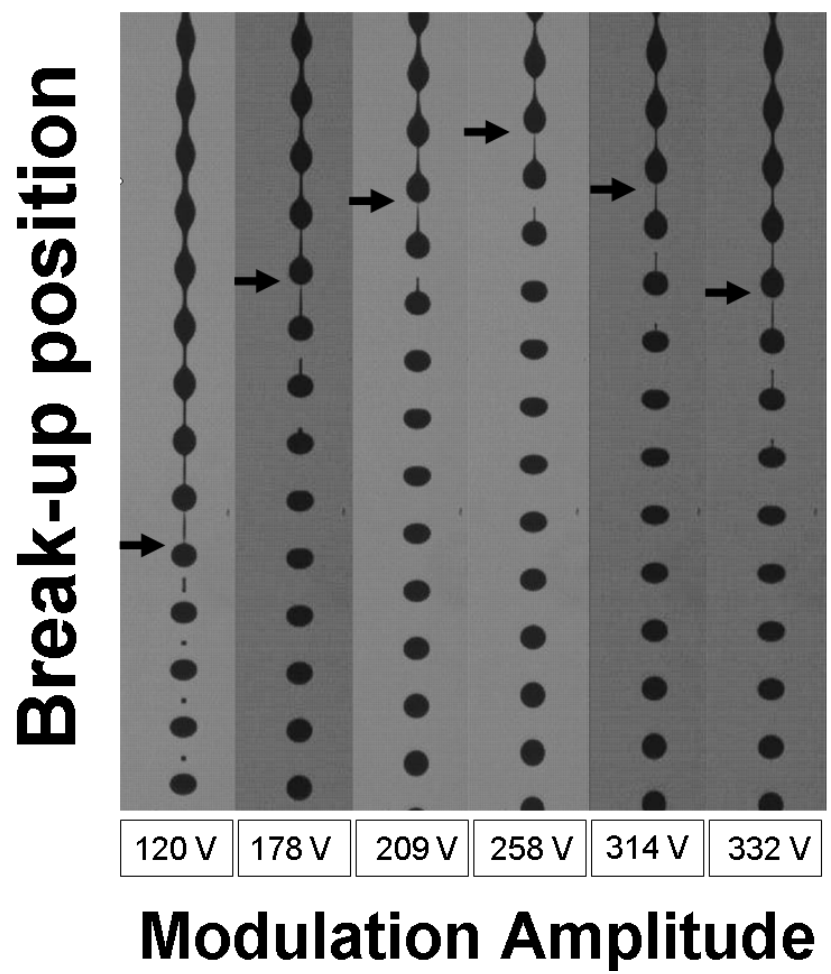

Figure 12 Shadowgraph imaging of a modulated jet of $50 \mu \mathrm{m}$ diameter. 
This technology is often used in the printing of caducity dates on plastic bottles and processed food containers. The printer was setup to jet a methyl ethyl ketone (MEK) based ink, at its standard modulation frequency of 64 $\mathrm{kHz}$ and a jet speed of $20 \mathrm{~m} / \mathrm{s}$.Stroboscopic methods are ideal and preferred in arrangements with a characteristic frequency or for a process that involves highly repeatable events. The basic principle is to illuminate the system in phase with the periodic phenomenon. This approach is applicable to a CIJ as the production of droplets is periodic and determined by the frequency of forcing. In this scheme, the exposure of the sensor can be arranged in such away that single or multiple flashes can be recorded.

The shadowgraph stroboscopic system was built around the printhead to observe the jet behaviour. The imaging setup consisted of a spark flash system with a flash duration of $20 \mathrm{~ns}$, a focusing system and an optical diffuser to produce a uniform short duration light background. The jet was visualized by a Navitar 12x ultra zoom microscope lens coupled to a CCD Prosilica (EC1380) camera with a resolution of 152.4 pixels $/ \mathrm{mm}$. The microscope lens was set to a depth of field of $\gg 200 \mu \mathrm{m}$ which is large enough to contain the whole volume of the jet. The CCD camera and the spark flash were continuously triggered in phase with the printing frequency to obtain stroboscopic images; the recorded images are the result of the stroboscopic superposition of approximately 10 flashes.

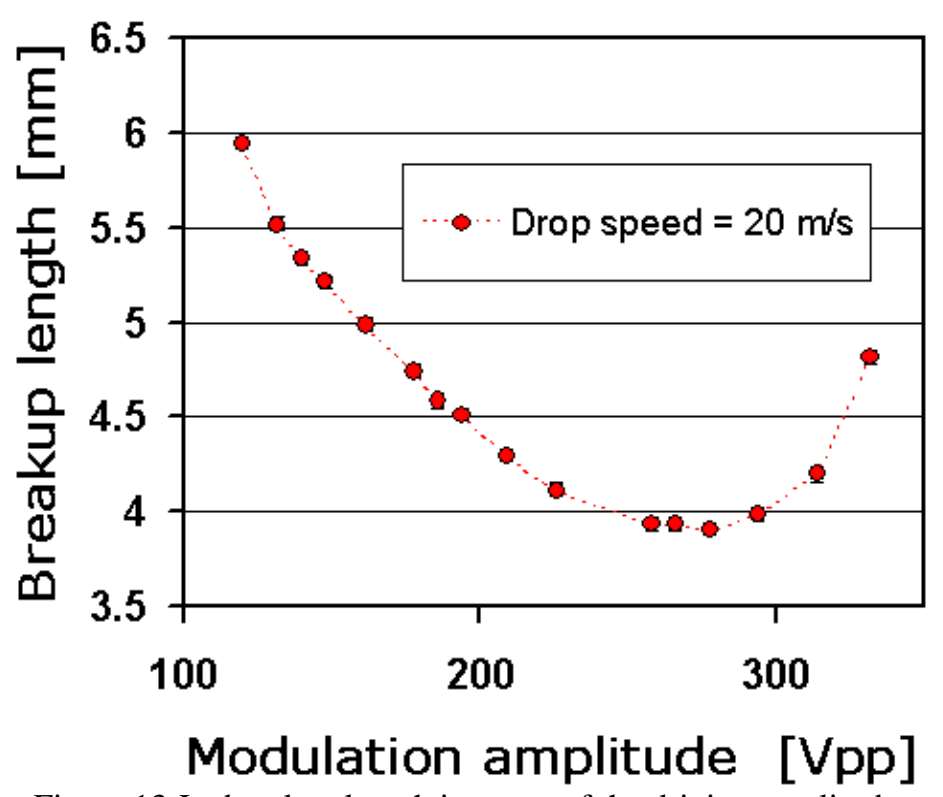

Figure 13 Jet breakup length in terms of the driving amplitude. 
Figure 12 shows some examples of shadowgraph images in terms of the modulation voltage used to drive the piezoelectric elements in the printhead. In a printhead, the drive modulation is delivered to the jet by a piezoelectric actuator located inside the printhead behind the nozzle. As the properties of both the piezoelectric element and the nozzle vary between suppliers, the optimisation of the jetting conditions is usually an empirical process that is carried out for each individual printhead. The jet break-up length, defined as the distance from the nozzle plane to the position of first break-up of the jet, is of commercial interest as this ultimately determines where the charging electrode is located (Curry and Portig, 1977). In the CIJ industry, shadowgraph images like the ones presented in Fig. 10 are used to determine the breakup length of the jet in terms of the modulation drive voltage to produce a calibration curve. These calibrations are then utilized to find the optimum distance for the electrode. The breakup curve obtained by shadowgraphy using the MEK-based ink and the Domino printhead is shown in Figure 13. The ideal point to set the printer is at the conditions producing the minimum breakup length because the jet is at it shortest length and so less susceptible to changes in environmental conditions. At this condition, the jet also exposes less surface, reduces evaporation of its components, has a minimum cross section and consequently has a minimum aerodynamic drag. The calibration curves can be derived automatically by the use of image analysis algorithms. The following section explains these techniques briefly.

\subsection{Shadowgraphy and image analysis methods}

The aim of this section is to briefly introduce some concepts of image analysis of shadowgraph digital pictures. Image analysis is a branch of computer science that deals with the detection of objects, still or in motion, from digital images and films (O'Gorman et al, 2008). This technology is currently used extensively in the study of fluid dynamics; some common examples are velocimetry in flows by particle tracking, droplet directionality by drop detection and ligament size distribution studies in sprays, (Adrian, 1991, Hutchings et al, 2007 and Castrejón-García et al, 2003).

Most common algorithms for image analysis detect the boundary of objects by identifying local changes of contrast or colour levels in an image. In shadowgraphy, sudden changes of contrast only occur in the vicinity of abrupt changes of refractive index which are usually localized at the ob- 
ject's boundary (as seen in Figure 14). As a consequence and as previously mentioned in Section 2, the shadowgraph technique is ideal for the use of image analysis.

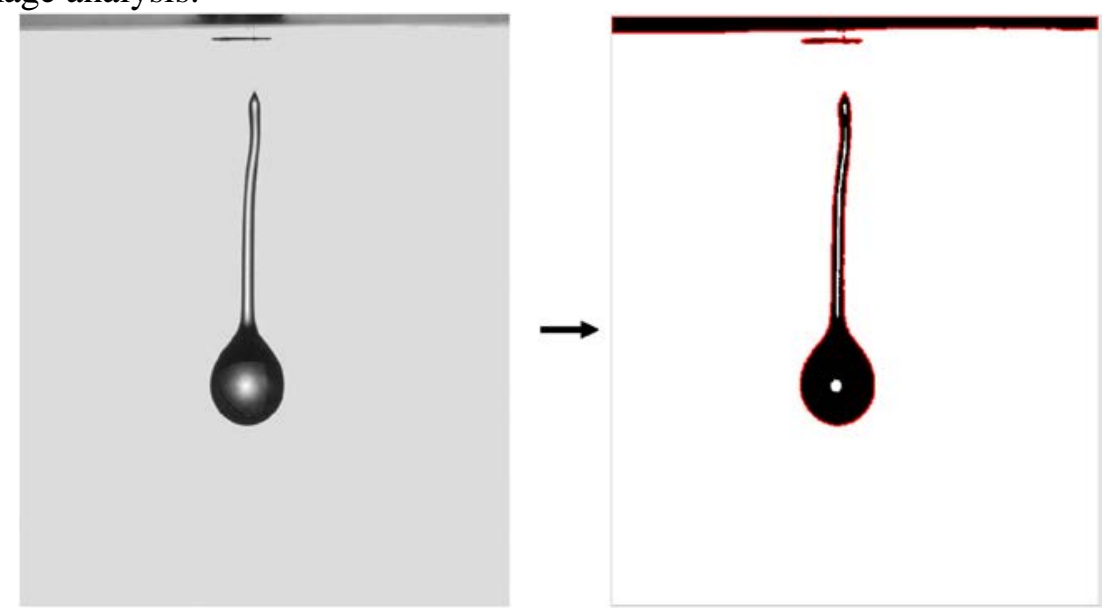

Figure 14 Boundary detection (in red) by image analysis.

Simple algorithms for boundary detection aim to convert colour images into black and white format where the object in study appears in black pixels and its background in white. In this type of algorithm, an image is first transformed into a grey-scale format so its pixel intensity levels can be discriminated by a threshold (RGB colour images are converted to grey scale by eliminating the hue and saturation information). In this way, the pixel intensity is individually compared with a pre-set intensity threshold. Any pixel of which the intensity level is above the threshold is converted to black while pixels in which the intensity is below the threshold are converted to white.

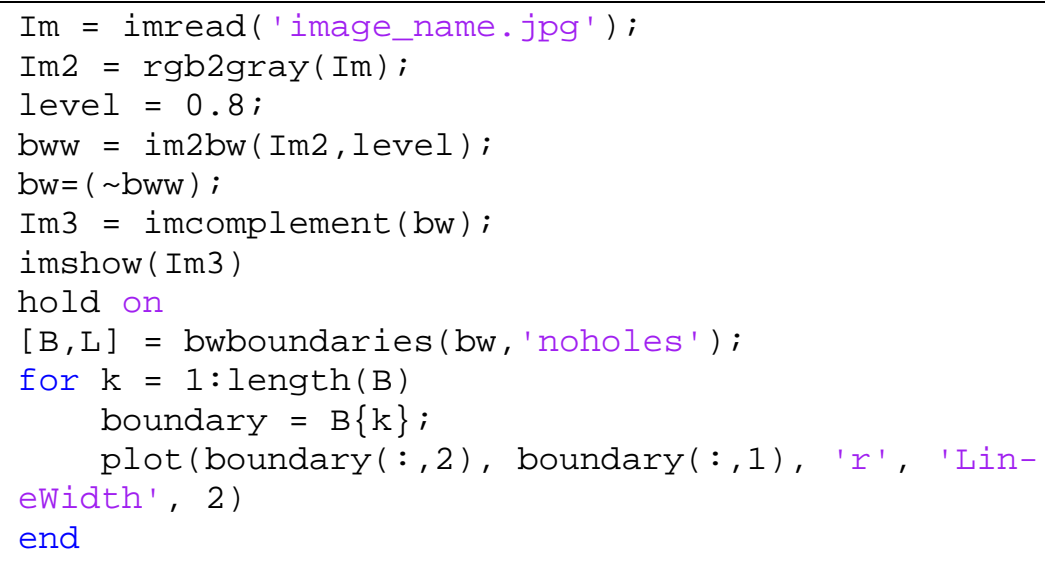


High speed shadowgraphy for the study of liquid drops 19

Figure 15 Basic algorithm (Matlab code) used to detect fluid boundaries. This code, without alterations, was used to produce Figure 12.

The object's boundary is then detected as the outer region of the black region. An example of this type of algorithm is shown in Figure 15. In the code presented in Fig 15, the image file is first loaded (line 1) and then converted into a grey-scale intensity image (line 2). The threshold level is set in line 3, line 4 compares it with the image pixels and line 5 records the resulting black and white (or binary) image (a value of 0 for black and 1 for white). The rest of the algorithm draws the image output as shown on the right in Figure 14. Once the detection of boundaries and objects is performed many different studies can be carried out, e.g. the area, the center of mass, and volumes of objects can be measured (Hutchings, 2007). These algorithms can also be used to obtain fluid properties such as surface tension or viscosity by analyzing the oscillations of droplets or the profile of continuous jets, (Bellizia et al, 2003 and Castrejón-García et al, 2011).

\section{Conclusions}

A brief review of the shadowgraph technique has been presented in this paper. A summary of the basic concepts to design and construct useful visualisation systems and some examples have been presented. Although the shadowgraph is a rather old technique, it is still in use in laboratories around the world in conjunction with image analysis techniques. Shadowgraph systems are nowadays used as a quantitative and precise technique, no longer as a pure visualisation mean, and survive among modern competitors such as particle image velocimetry and laser Doppler anemometry. Further advances in electronics, optics, photography, and sensors development can only strengthen the place of shadowgraphy as a very useful technique in the study of fluid dynamics and objects in motion.

\section{Acknowlegments}

This work was partially supported by the UK EPSRC and industrial partners in the Innovation in Industrial Inkjet Technology project. JRCP acknowledges the support from the Grupo Santander Academic Travel Fund of the University of Cambridge. The authors are grateful for the assistance of J. Waldmeyer during the recording of the CIJ stroboscopic images. 


\section{References}

Adrian R J, (1991) Annu. Rev. Fluid Mech. 23, 261-304.

Bellizia G, Megaridis G M, Mc Nallan M and Wallace D V, (2003) Proc. R. Soc. Lond. A 459, 2195-2214.

Bruce C A, (1976) IBM J. Res. Dev. 20, 258-270.

Castrejón-García R, Castrejon-Pita J R, Martin G and Hutchings I M., (2011) Rev. Mex. Fís. 57(3), 266-275.

Castrejón-Pita J R, et al., (2011) Biomicrofluidics 5, 014112.

Castrejón-Pita J R, Martin G, Hoath S and Hutchings I M, (2008) Rev. Sci. Instrum. 79, 075108.

Castrejón-García R, Sarmiento-Galán A, Castrejón-Pita J R and Castrejón-Pita A A, (2003) Fractals 11, 155-161.

Castrejón-García R and Milan J, (1982) Application of the shadowgraph technique to the analysis of mechanical sprayers, IX International Meeting on Boilers and Pressure Vessels (AMIME), Cuernavaca, Mexico.

Curry S A and Portig H, (1976) IBM J. Res. Dev. 21, 10-20.

Eggers J and Villermaux E (2008) Rep. Prog. Phys. 71, 036601.

Highton S, (2011) Virtual Reality Photography: Creating Panoramic and Object Images, Library of Congress, ISBN: 978-0-165-34223-8, p 38.

Hutchings I M, Martin G D and Hoath S D, (2007) Journal of Imaging Science and Technology 51(5), 438-444.

Jones A R, (1977) Progress in Energy and Combustion Sciences 3, 225-234.

Kalaali A, Lopez B, Attane P and Soucemarianadin A, (2003) Physics of Fluids 15, 2469-2479.

Lambrecht R W, and Woodhouse C, (2011) Way Beyond Monochrome, 2nd Ed. Focal Press, Elsevier, pp 134-136.

Le Moyne L, Freire V and Conde D Q, (2008) Chaos Solitons \& Fractals 38(3), 696-704.

Martin G D, Hoath, S D and Hutchings I M, (2007) Journal of Physics: Conference Series 105, 01200

O'Gorman L, Sammon M J and Seul M, (2008) Practical Algorithms for Image Analysis, Cambridge University Press, Cambridge UK.

Savart F, (1833) Ann. Chim. 53, 337-386.

Strutt J W (Lord Rayleigh), (1896) The Theory of Sound, Volume II. Macmillan and Co. ltd, New York. 